\title{
Germ-line intrachromosomal recombination restores fertility in transgenic MyK-103 male mice
}

\author{
Thomas M. Wilkie, ${ }^{1}$ Robert E. Braun, ${ }^{2,4}$ Walter J. Ehrman, ${ }^{3}$ Richard D. Palmiter, ${ }^{2}$ and Robert E. \\ Hammer $^{3}$ \\ ${ }^{1}$ Biology Division, California Institute of Technology, Pasadena, California 91125; ${ }^{2}$ Howard Hughes Medical Institute, \\ Department of Biochemistry, University of Washington, Seattle, Washington 98195; ${ }^{3}$ Howard Hughes Medical Institute, \\ Department of Biochemistry, University of Texas Southwestern Medical Center, Dallas, Texas 75235
}

\begin{abstract}
Males of the MyK-103 line of transgenic mice are fertile and sire litters of normal size, but they never transmit the transgene, whereas females transmit the transgene with normal frequency. The chromosome originally bearing the transgene can be transmitted through the male germ line, but only after the transgene is deleted or rearranged by intrachromosomal recombination. The transgene encodes a functional herpes simplex virus (HSV) thymidine kinase gene that causes sperm infertility when expressed in postmeiotic germ cells. Immunocytochemistry revealed clones of germ cells that fail to express HSV thymidine kinase. We postulate that these cells arose by transgene deletion in embryonic germ cells and postnatal spermatogonial stem cells and that they are responsible for the normal fertility of MyK-103 males. The frequency of recombination events at the integration locus suggests that it contains a hotspot for mitotic recombination.
\end{abstract}

[Key Words: recombinational hotspot; spermatogonial stem cell; transmission ratio distortion]

Received August 30, 1990; revised version accepted November 16, 1990.

The MyK-103 pedigree has a unique pattern of male germ-line transmission (Palmiter et al. 1984). Males are fertile in natural matings but never transmit the integrant to their offspring. The block to germ-line transmission in MyK-103 males occurs prior to fertilization because males and females both have litters of normal size, but only females transmit the integrant to roughly half of their offspring. This pattern of germ-line transmission appears similar to the cases of transmission ratio distortion that have been studied at the mouse $t$ complex and the Drosophila segregation distorter locus (Bennett 1975; Hartl and Hiraizumi 1976). In these cases, however, the germ-line transmission of one allele has a selective advantage over the other of up to $99 \%$, but it is not absolute, as in MyK-103 males.

Initially, we proposed that transmission ratio distortion in MyK-103 males resulted from a selective disadvantage of sperm containing the transgene due to integration near a gene that was necessary for the formation of fertile spermatozoa (Palmiter et al. 1984). The observation that there was a depletion of transgenic sperm in the cauda epididymus of some males supported this view. A complication of this interpretation is that all the descendants of a spermatogonial stem cell are connected by intercellular bridges during premeiotic divisions, meiosis, and postmeiotic maturation. These bridges are

"Present address: Department of Genetics, University of Washington, Seattle, Washington 98195 USA. about $1 \mu \mathrm{m}$ in diameter (Dym and Fawcett 1971) and would, in principle, allow sharing of macromolecules among all the cells in the clone. Thus, to account for transmission ratio distortion in MyK-103 males as due to insertional inactivation of a gene critical for spermatogenesis, we would also have to propose that development of the haploid transgenic spermatids could not be rescued by their meiotic partners bearing wild-type alleles.

We subsequently cloned the integration site of the transgene and found that it was flanked by a $5-\mathrm{kb}$ duplication of DNA that was present only once at the preintegration site on chromosome 6 (Wilkie and Palmiter 1987). This observation suggested the possibility that the transgene, which contains a functional copy of the metallothionein/herpes simplex virus (HSV) thymidine kinase fusion gene (pMK), might frequently be lost by intrachromosomal recombination through the duplicated sequences. If the transgene, instead of resulting in insertional inactivation of an essential gene, resulted in expression of a gene product that caused sperm infertility, then the transgenic males would be sterile due to sharing of gene products among spermatids. However, recombination events that deleted the transgene could result in clones of wild-type spermatids that might develop normally and account for the fertility of the MyK-103 males.

This paper provides both genetic and immunocyto- 
chemical data supporting the latter view. We used a restriction fragment length polymorphism (RFLP) within the $5-\mathrm{kb}$ duplicated sequence that allowed us to distinguish male germ-line transmission of the chromosome bearing the transgene independently of the transgene itself. We show that deletion of the MyK-103 transgene by homologous recombination occurs at a high frequency in embryonic germ cells and mitotically active spermatogonial stem cells, resulting in the equal transmission of the recombinant MyK-103 chromosome and its meiotic partner. The evidence presented here, and independently by Braun et al. $(1989,1990)$, shows that macromolecules can be shared among syncytial spermatids and that postmeiotic expression of HSV thymidine kinase causes sperm infertility. The synthesis of these observations renders a satisfying explanation of the transmission distortion in MyK-103 males, one of the oldest lines of transgenic mice in existence (Palmiter et al. 1982).

\section{Results}

Homologous recombination in the male germ line deletes the transgene

The structure of the MyK-103 integrant is shown in Figure 1. Genetic instability of this integrant could result from deletion by intrachromosomal recombination between the flanking duplicated mouse sequence. We would not have detected male germ-line transmission of the hypothetical recombinant chromosome in the MyK-103 pedigree because it would be indistinguishable from the nontransgenic homolog in mice of the original C57BL/6 $\times$ SJL background. To test this hypothesis, we used a RFLP resulting from a $1.5-\mathrm{kb}$ deletion in AKR inbred mice to follow germ-line transmission of the

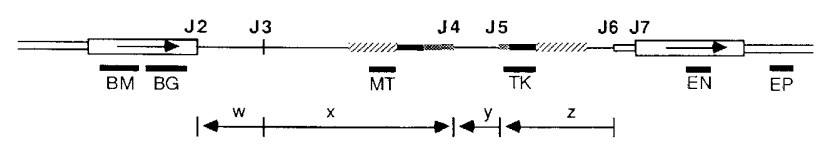

Figure 1. Structure of the MyK-103 integrant. The integrant is composed of $19 \mathrm{~kb}$ of DNA flanked on either side by a $5-\mathrm{kb}$ duplication of mouse DNA that is normally found in single copy at this locus. The integrant contains four fragments of the plasmid $\mathrm{pMK}(\mathrm{w}, \mathrm{x}, \mathrm{y}$, and $z)$. The sequences in $\mathrm{pMK}$ include pBR322 (thin line), the metallothionein I promoter (hatched box), the HSV thymidine kinase coding sequence (thick black line), and downstream sequence (shaded line; for further details, see Wilkie and Palmiter 1987). Note that the duplicated flanking sequences (box, arrow inclusive) are in the same relative orientation, as are the repeated DNA sequences of pBR322 present in the pMK fragments marked $w, y$, and $z$. The novel junctions formed by ligation of pMK fragments are marked $\mathrm{J} 2$ through J6. A 532-bp fragment of mouse repetitive DNA is positioned between J6 and J7 (Wilkie and Palmiter 1987). The origin of the restriction fragments that were used to prepare hybridization probes is indicated: (BM) $1.5 \mathrm{~kb} \mathrm{BamHI}$ (BG) $1.6 \mathrm{~kb}$ BglII; (MT) $0.6 \mathrm{~kb} \mathrm{StuI-BglII;} \mathrm{(TK)} 2.0 \mathrm{~kb} \mathrm{BgIII-SmaI;} \mathrm{(EN)} 0.9$ $\mathrm{kb}$ EcoRI-NcoI; and (EP) $0.6 \mathrm{~kb} E c o \mathrm{RI}-P v u I I$. The sequences of all probes except EP occur twice in the MyK-103 insert. (Diagram adapted from Wilkie and Palmiter 1987.)

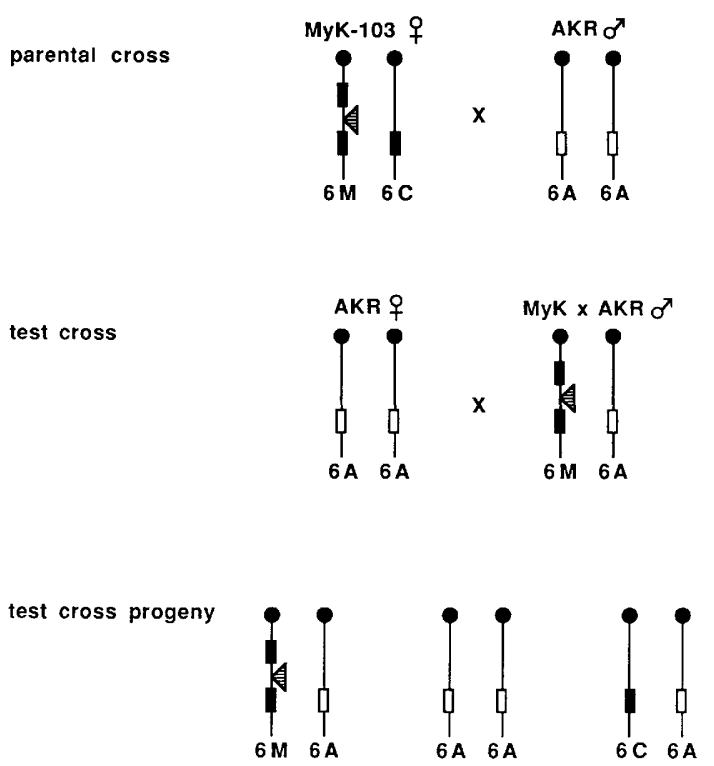

Figure 2. MyK-103 $\times$ AKR test-cross. MyK-103 mice are heterozygous at the integration locus; one homolog $(6 \mathrm{M})$ contains the prototype MyK-103 insert and is flanked by the duplicated sequence, and the other homolog $(6 \mathrm{C})$ contains one copy of this sequence, as do C57BL/6 and SJL mice. AKR mice are homozygous for a small deletion (6A) within the sequence that was duplicated in the $6 \mathrm{M}$ allele.

transgenic chromosome independently of the transgene. The RFLP maps to the proximal region of chromosome 6 and lies $2.5 \mathrm{~kb}$ from the integration site in MyK-103 mice (Wilkie and Palmiter 1987). By virtue of this small deletion, the AKR allele on chromosome $6(6 \mathrm{~A})$ could be distinguished from either the allele containing the pMK insert $(6 \mathrm{M})$ or the nontransgenic allele $(6 \mathrm{C})$ carried in MyK-103 mice. As diagramed in Figure 2, the 6A allele was introduced into the MyK-103 line by crossing AKR males $(6 \mathrm{~A} / 6 \mathrm{~A})$ to heterozygous MyK-103 females (6M/6C). Transgenic male offspring that inherited the $6 \mathrm{~A}$ allele from their father and the $6 \mathrm{M}$ allele from their mother were chosen as test-cross males to be mated with AKR females. Considering all possibilities, the test-cross males $(6 \mathrm{~A} / 6 \mathrm{M})$ might transmit the $6 \mathrm{~A}$ or $6 \mathrm{M}$ allele, as well as the $6 \mathrm{C}$ allele, which could be generated from the $6 \mathrm{M}$ allele by homologous recombination.

Segregation of the $6 \mathrm{~A}, 6 \mathrm{M}$, and the recombinant $6 \mathrm{C}$ alleles was assayed in the test-cross progeny by Southern blot or dot hybridization of tail DNA. The Southern blot shown in Figure 3B compares the hybridization patterns of genomic DNA isolated from parental mice, test-cross males, and test-cross progeny. DNA was digested with BamHI and hybridized with the E-N probe (Fig. 3A), which maps completely within the deletion in the $6 \mathrm{~A}$ allele. MyK-103 DNA (6M/6C) yielded two bands $(6.5$ and $5.5 \mathrm{~kb}$ ) that hybridized to the E-N probe. The $5.5 \mathrm{~kb}$ band is twice the intensity of the $6.5-\mathrm{kb}$ band because the former is found in both the $6 \mathrm{M}$ and $6 \mathrm{C}$ alleles, whereas the latter is derived only from the $6 \mathrm{M}$ allele. DNA from AKR mice $(6 \mathrm{~A} / 6 \mathrm{~A})$ did not hybridize, as expected, and DNA from test-cross males $(6 \mathrm{M} / 6 \mathrm{~A})$ yielded 

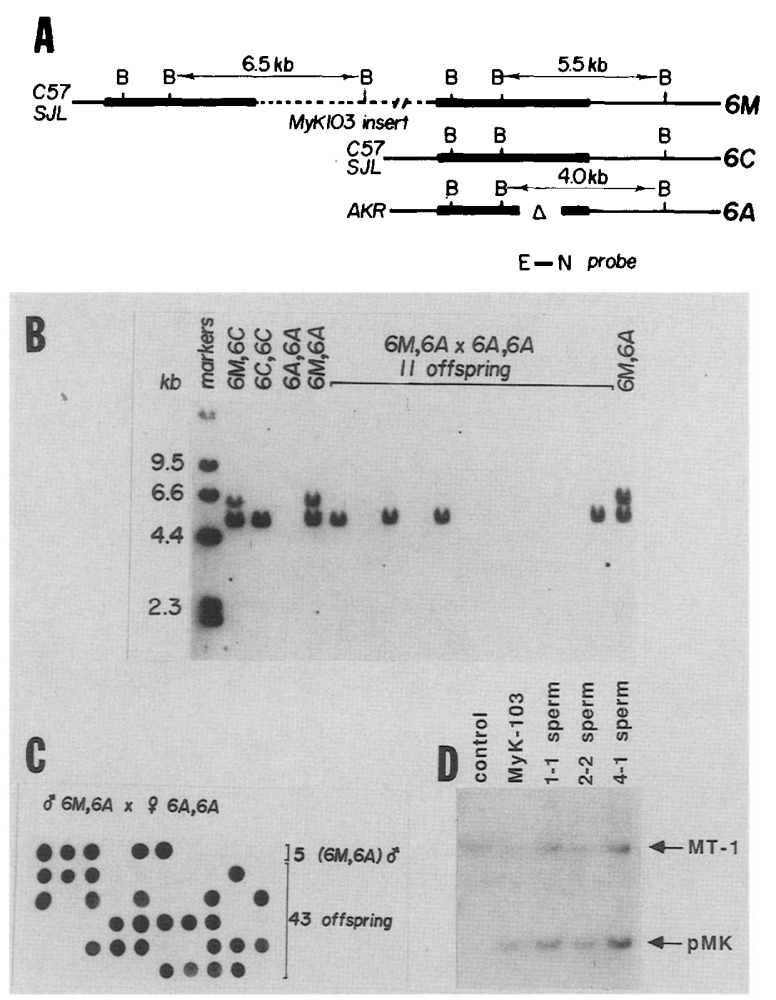

Figure 3. Only germ cells that deleted the HSV thymidine kinase gene produce fertile sperm. $(A)$ Location of the BamHI (B) restriction sites and the DNA fragments (in kilobases) that hybridize to the EN probe. The EN restriction fragment lies entirely within the breakpoints of the AKR deletion. $(B)$ Southern blot of liver DNA $(5 \mu \mathrm{g})$ from parental mice (MyK-103: 6M,6C; AKR: $6 \mathrm{~A}, 6 \mathrm{~A})$, test-cross males $(6 \mathrm{M}, 6 \mathrm{~A})$, and fetal DNA $(5 \mu \mathrm{g})$ from 11 test-cross progeny $(6 \mathrm{M}, 6 \mathrm{~A} \times 6 \mathrm{~A}, 6 \mathrm{~A})$. DNA was cut with $B a m H I$ and hybridized with the EN probe. $|C|$ Dot hybridization of tail DNA $(10 \mu \mathrm{g})$ from five test-cross males and fetal DNA from 43 test-cross progeny was hybridized with the EN probe; 24 offspring inherited the $6 \mathrm{C}$ allele and 19 offspring inherited the $6 \mathrm{~A}$ allele from their fathers. $(D)$ Sperm DNA $(5 \mu \mathrm{g})$ was isolated from the cauda epididymides of three test-cross males (1-1, 2-2, and 4-1; 8 weeks of age). Somatic DNAs (spleen; $5 \mu \mathrm{g}$, isolated from a nontransgenic littermate and a MyK-103 hemizygous female, were included as controls. DNA was cut with Pst and hybridized with the MT probe (Fig. 1) which identified both the transgene $(\mathrm{pMK}, 3.5 \mathrm{~kb})$ and the endogenous metallothionein I gene (MT-I, 6.6 kb; Palmiter et al. 1984). Hybridization to MT-I served a dual purpose as an internal control for the amount of DNA loaded in each lane and as a relative measure of pMK in spleen and sperm DNA. The relative intensities of the pMK and MT-I bands in spleen and sperm were equivalent, indicating that nearly half of the sperm produced by these young test-cross males inherited HSV thymidine kinase.

the $5.5-\mathrm{kb}$ and $6.5-\mathrm{kb}$ bands at equal intensity because only the $6 \mathrm{M}$ allele hybridized to the E-N probe. This blot also shows that when 11 test cross progeny were assayed, seven offspring inherited the $6 \mathrm{~A}$ allele and four offspring inherited the $6 \mathrm{C}$ allele from their father. In total, 54 progeny sired by five test-cross males were assayed by dot hybridization, first with the E-N probe (Fig.
3C) and, after this probe was removed from the filter, with pBR322 (data not shown). None of the progeny inherited the $6 \mathrm{M}$ allele, consistent with the observation that MyK-103 males never transmit the prototype MyK-103 insert. The number of test-cross progeny that inherited the $6 \mathrm{~A}$ or $6 \mathrm{C}$ allele was nearly equal; 26 progeny inherited the $6 \mathrm{~A}$ allele and the remaining 28 progeny inherited the recombinant $6 \mathrm{C}$ allele from their fathers. A Southern blot of sperm DNA from three of these test-cross males that was hybridized with the metallothionein I (MT-I) probe showed that roughly half of their sperm carried the transgene (Fig. 3D). Thus, the vast majority of sperm produced by the test-cross males carried the $6 \mathrm{M}$ allele or its $6 \mathrm{~A}$ meiotic partner, yet the recombinant $6 \mathrm{C}$ allele was transmitted at essentially equal frequency with the $6 \mathrm{~A}$ allele. Therefore, sperm that carried the transgenic $6 \mathrm{M}$ allele or its $6 \mathrm{~A}$ meiotic partner were infertile. The only fertile sperm that were produced by the test-cross males carried the recombinant $6 \mathrm{C}$ allele or its $6 \mathrm{~A}$ meiotic partner and, relative to the transgenic sperm, were few in number.

\section{Mosaic expression of HSV thymidine kinase in MyK-103 testes}

The genetics indicated that the MyK-103 testis was a mosaic that contained both transgenic and recombinant nontransgenic germ cells. To visualize this mosaicism, we took advantage of the fact that HSV thymidine kinase mRNA was readily detected in testes (Fig. 4A) and the protein could be visualized by immunocytochemistry.

In MyK-103 males, HSV thymidine kinase expression in the testis appears to be restricted to round and elongating spermatids, with the heaviest staining in the latter cell type (Fig. 5B). The immunocytochemical assay did not detect HSV thymidine kinase protein in the testes of nontransgenic littermates (Fig. 5A). In the six MyK-103 hemizygous males that were assayed, some seminiferous tubules expressed HSV thymidine kinase in all spermatids. Considering that only half of the spermatids inherited the pMK transgene following meiosis in a hemizygous male, it is striking that even those spermatids that inherited the nontransgenic homolog of chromosome 6 contained HSV thymidine kinase protein. The equal distribution of HSV thymidine kinase between genetically distinct spermatids was presumably accomplished by movement of the mRNA, and perhaps protein as well, across the intercellular bridges in a syncytial cluster of spermatids (Braun et al. 1989, 1990). Thus, all spermatids developing within a syncytium that expressed HSV thymidine kinase were phenotypically transgenic.

Male sterility has recently been correlated with testicular expression of HSV thymidine kinase in several different lines of transgenic mice (Al-Shawi et al. 1988; Iwakura et al. 1988; Heyman et al. 1989). In the most definitive study, all lines that expressed relatively high levels of HSV thymidine kinase in the spermatids of hemizygous mice were sterile, whereas low-level expres- 


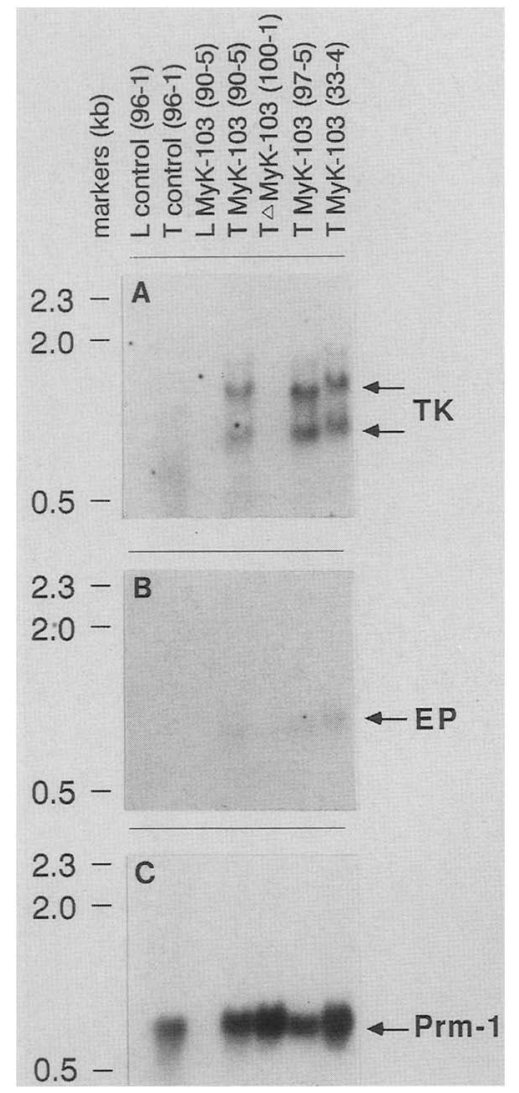

Figure 4. Northern analysis of MyK-103 testis RNA. Total RNA (10 $\mu \mathrm{g})$ was isolated from either liver $(\mathrm{L})$ or testis $(\mathrm{T})$ from five adult males and hybridized with a probe specific to one of three probes. $(A) \mathrm{HSV}$ thymidine kinase (TK). $(B)$ The EP restriction fragment. (C) A 0.6-kb EcoRI fragment including coding sequence of the mouse protamine 1 gene (Prm-l; Peschon et al. 1987). Male 96-1 was a nontransgenic offspring in the MyK-103 pedigree, males 90-5, 97-5, and 33-4 inherited the prototype MyK-103 insert, and male 100-1 inherited the rearranged transgene $\Delta \mathrm{MyK}$.

sion did not affect male germ-line transmission of the HSV thymidine kinase gene (Braun et al. 1990). Immunocytochemical analysis of HSV thymidine kinase expression levels in the round and elongating spermatids of these transgenic mice was compared to expression in MyK-103 spermatids. The intensity of staining in MyK-103 testes was similar to that observed in testes from the sterile transgenic males (data not shown). Some other unusual features seen in the sterile mice, such as multinucleated giant cells in the ducts of the cauda epididymis (Braun et al. 1990), were also found in some MyK-103 males. These similarities indicated that HSV thymidine kinase expression in MyK-103 spermatids contributes to, and may be the sole cause of, infertility of transgenic sperm and their meiotic partners. However, MyK-103 males are fertile because a significant fraction of their spermatid syncytia do not express HSV thymidine kinase.

The contrast between syncytia that did or did not ex- press HSV thymidine kinase elicited intricate mosaic patterns in antibody-stained cross sections of MyK-103 testes. Some of the seminiferous tubules that were revealed by cross section did not express HSV thymidine kinase in any of their spermatids (data not shown). The absence of expression in certain tubules did not simply reflect developmental regulation of HSV thymidine kinase translation because elongating spermatids are present in all seminiferous tubules and elongating spermatids in each stage of development could express HSV thymidine kinase. Other seminiferous tubules appeared sectored by distinct patches of spermatids that did not express HSV thymidine kinase (Fig. 5B, tubule 1). Juxtaposition of two patches of spermatids within a single tubule defines the boundary of at least two syncytial clusters of spermatids. Sectoring was also observed in the mosaic tubule shown in Figure 5D where the elongating spermatids expressed HSV thymidine kinase, but it was not expressed in the underlying round spermatids. A similar pattern was apparent in the tubules marked $2-5$ in Figure 5B. Elongating and round spermatids develop in separate syncytia, prohibiting the diffusion of HSV thymidine kinase between these two cell types. The elongating spermatids that expressed HSV thymidine kinase were descendents of spermatogonia that committed to spermatogenesis in the seminiferous epithelium one cycle earlier than the underlying round spermatids within the same tubule.

On the basis of our immunocytochemical analysis, we can refine the interpretation of the genetic results presented above. We infer that the transgenic sperm and their meiotic partners which were infertile in MyK-103 males developed from syncytial germ cells that expressed HSV thymidine kinase. The spermatids that did not express HSV thymidine kinase were the descendents of spermatogonial stem cells which, at some time during their development and regenerative divisions, deleted the pMK transgene via homologous recombination (Fig. 6). Those spermatogonial stem cells in mosaic MyK-103 testes that had deleted pMK produced fertile sperm.

\section{Frequency of homologous recombination in the MyK-103 male germ line}

Testicular mosaicism is a characteristic of MyK-103 males that was never observed in other hemizygous transgenic males that expressed HSV thymidine kinase in spermatids (Braun et al. 1990). Mosaic patterns of expression among spermatids was observed in all six hemizygous MyK-103 males that were assayed. The extent of mosaicism ranged from $5 \%$ to $80 \%$ of the seminiferous tubules which contained nontransgenic spermatid syncytia, and older mice had a greater proportion of nontransgenic spermatids than younger mice.

It is possible to estimate the deletion frequency of pMK per cell division in the MyK-103 male germ line given the age of the mouse when sacrificed and the percentage of nontransgenic spermatids, in addition to certain assumptions (see Hartl 1971). The assumptions are (1) the deletion frequency was constant throughout em- 
Wilkie et al.

Figure 5. Immunocytochemical analysis of HSV thymidine kinase expression. Testes from adult animals were fixed, sectioned, and stained with primary and secondary antibodies as described in Materials and methods. (A) Control testis; magnification, $90 \times$. (B) Hemizygous MyK-103 testis (92-3) 11 weeks of age; magnification, $62 \times$. (C) Hemizygous $\triangle$ MyK testis; magnification, $90 \times$. (D) 92-3 testis, counterstained with hematoxylin; magnification, $360 \times$. Expression of HSV thymidine kinase was restricted to round and elongating spermatids.
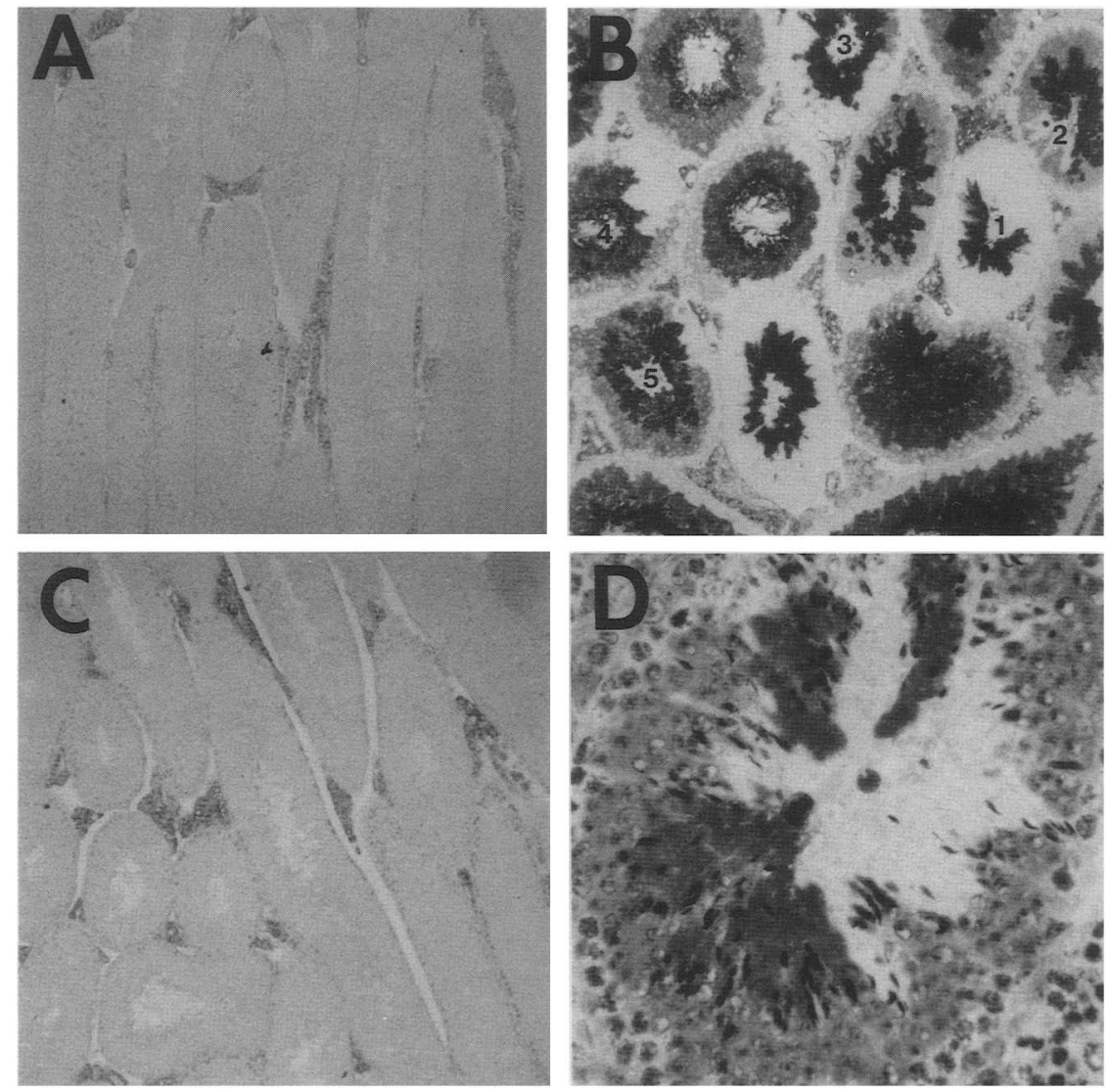

bryonic and postnatal life; (2) there was no selective pressure on nontransgenic cells during proliferation of the primordial germ cells and prospermatogonia, or during replication of spermatogonia stem cells and subsequent commitment to spermatogenesis; (3) the proportion of nontransgenic spermatids that we determined by immunocytochemistry was equal to the proportion of nontransgenic spermatogonial stem cells. Given these assumptions, the germ-line deletion frequency in a male sacrificed at 11 weeks of age, which was about $5 \%$ mosaic, was estimated to be $1.8 \times 10^{-3}$ per cell division (see Materials and methods). The frequency estimated from a second mouse (sacrificed at 87 weeks, $25 \%$ mosaic) was $2.5 \times 10^{-3}$ per cell division. Consistent with the high frequency of transgene deletion, the production of transgenic sperm in MyK-103 males has been shown to diminish with age (T.M. Wilkie and C. Muller, unpubl.).

\section{A novel transcript in MyK-103 testis}

In addition to HSV thymidine kinase, MyK-103 testes also expressed another transcript that was not observed in nontransgenic siblings. To identify this transcript we screened restriction fragments of mouse genomic DNA that were isolated from $\lambda$ clones that spanned either flank of the pMK insert ( $\lambda 2$ and $\lambda 21$; see Wilkie and Palmiter 1987). Northern blots of poly(A) RNA isolated from MyK-103 and nontransgenic testes were hybridized with the various restriction fragments. The hybridization probe EP identified an 800-nucleotide transcript specifically in MyK-103 testes (Fig. 4B). This probe was isolated from mouse DNA to the right of the $\mathrm{pMK}$ insert (Fig. 1) and is present in single copy per haploid genome; it does not cross-hybridize with the transgene $\mathrm{pMK}$ or to mouse repeat DNA. To assay the normal expression pattern of this transcript, the EP probe was hybridyzed to Northern blots of RNA that was isolated from a variety of germ cell sources, including primitive type A spermatogonia, A and B spermatogonia, spermatocytes, and spermatids (Thomas et al. 1989). We also screened Northern blots of poly(A) RNA from adult somatic tissues, but the 800-nucleotide transcript has never been identified in any of these samples; it remains a unique feature of the MyK-103 testis. Expression of this aberrant transcript in testis might be enhanced by proximity of the MT-1 promoter in MyK-103 mice and it may contribute to the MyK-103 phenotype.

\section{Male germ-line transmission of rearranged $\mathrm{MyK}$-103 transgenes}

We have shown that sperm fertility is recovered when the entire transgene insert is deleted by recombination between the flanking duplicated mouse sequences. Fertile sperm might also be produced if the metallothionein/HSV thymidine kinase fusion genes were deleted 
Stem Cell

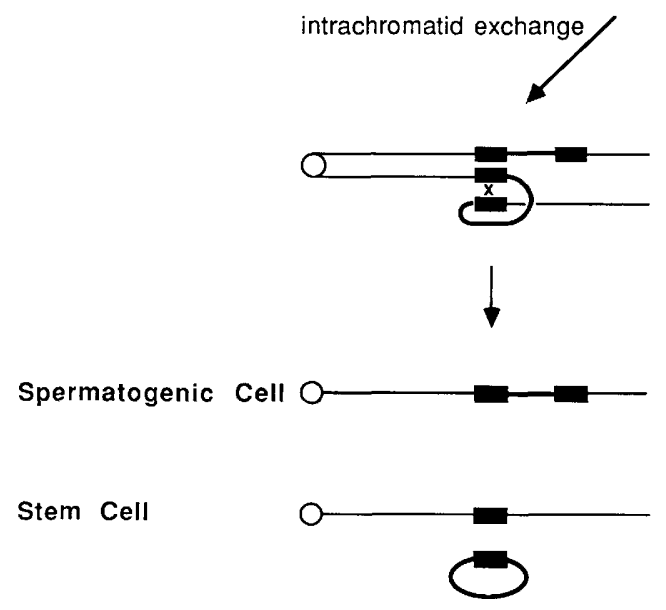

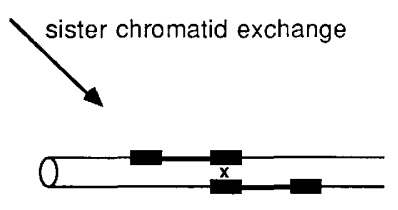

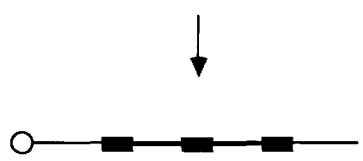

$\mathrm{O}$
Figure 6. Intrachromosomal recombination deletes pMK. Deletion of plasmid integrants similar in structure to the MyK-103 insert typically occurs by intrachromosomal recombination, either by intrachromatid or sister chromatid exchange (Jackson and Fink 1981). Recombination might occur any time during the cell cycle although the model depicts it occurring after DNA replication. As shown, the recombinant chromosome would be retained in the germ line if it were inherited by the self-renewing stem cells. The transgenic chromosome would be lost from this stem cell lineage if the daughter cell that inherited it committed to spermatogenesis. by recombination between duplicated sequences of pBR322 within the insert. Although hemizygous males never transmit the prototype MyK-103 insert to offspring, we have observed male germ-line transmission of two distinct types of transgene rearrangements. These rearrangements were distinguished from each other and the prototype MyK-103 insert by their restriction patterns on Southern blots. DNA from hemizygous mice containing the prototype MyK-103 insert produced five bands that hybridized to pBR322 when cut with PvuII, whereas the type 1 rearrangement $(\triangle \mathrm{MyK})$ produced a single 6.6-kb restriction fragment (Fig. 7A) and the type 2 rearrangement $(\Delta+\mathrm{TK})$ produced two bands of 8.5 and $4.5 \mathrm{~kb}$ in length (Fig. $7 \mathrm{~B}$ ).

The structure of the rearrangements inherited by $\Delta \mathrm{MyK}$ and $\Delta+\mathrm{TK}$ mice (Fig. 8 ) was determined by additional Southern blot analysis. For example, the TK probe hybridized to the 8.5- and 5.9-kb bands from MyK-103 mice but only to the 8.5 -kb band in $\Delta+\mathrm{TK}$ (Fig. 7C) and it did not hybridize to $\Delta \mathrm{MyK}$ (data not shown). It is clear that the $8.5-\mathrm{kb}$ PvuII restriction fragment in $\Delta+\mathrm{TK}$ corresponds to the right junction fragment spanning J5, J6, and $\mathrm{J7}$ in MyK-103; likewise, the 4.5-kb band, which only hybridizes to pBR322, corresponds to the left junction fragment that spans J2 (Wilkie and Palmiter 1987). To create $\Delta+\mathrm{TK}$, and thus delete the 5.9 -kb band, homologous recombination probably occurred between the DNA sequences that were in the same relative orientation surrounding the Pvull sites indicated in Figure 8.

The structure of the $\Delta \mathrm{MyK}$ transgene was determined following additional Southern blot analysis of DNA from a hemizygous mouse compared side by side with DNA from a nontransgenic control and a prototype MyK-103 transgenic mouse. Somatic DNA from each animal was cut with $B a m H I$ and EcoRI, in addition to $P_{v u I I}$ and hybridized on separate filters with either radiolabeled pBR322 or the restriction fragments $B G$ or BM (see Fig. 1). pBR322 hybridized to a single restriction fragment generated by digestion with PVuII $(6.6 \mathrm{~kb}$, see Fig. $7 \mathrm{~A})$, BamHI $(5.4 \mathrm{~kb})$, or EcoRI $(5.3 \mathrm{~kb})$; the BG and
BM probes hybridized to the expected fragments (data not shown). $\triangle \mathrm{MyK}$ apparently arose by homologous recombination between two copies of pBR322 that were also oriented in the same direction within the nucleotide sequences indicated in Figure 8.

In total, 7 of 44 fertile males in the MyK-103 pedigree transmitted rearranged transgenes. Each rearrangement apparently occurred independently because six of the seven males did not have the same mother (Table 1), and the two $G_{0}$ males that did have the same mother both inherited the prototype MyK-103 insert, as demonstrated by the hybridization pattern to somatic DNA (Fig. 7A and data not shown). This inheritance pattern also suggests that the rearrangements must have occurred de novo in the germ line of $G_{0}$ males. In contrast, the rearrangement carried in the $\Delta+$ TK line probably occurred in a female during embryogenesis, because both male $35-1$ and his brother $35-4$ inherited $\Delta+\mathrm{TK}$ from their mother (Fig. 7B; see Hartl 1971).

Given that $\Delta \mathrm{MyK}$ and $\Delta+\mathrm{TK}$ have retained some portion of pMK and the flanking duplicated sequences, it is apparent that the structure of the rearranged transgenes is very similar to the prototype MyK-103 insert. If transmission ratio distortion in MyK-103 males were caused by gene disruption, then $\Delta \mathrm{MyK}, \Delta+\mathrm{TK}$, and MyK-103 would most likely have similar phenotypes due to the overall similarity in structure between these three lines of mice. However, both the $\Delta \mathrm{MyK}$ and $\Delta+\mathrm{TK}$ inserts were transmitted through the germ line of hemizygous $\left(G_{1}\right)$ males without apparent bias (Table 1). HSV thymidine kinase protein could not be detected by immunocytochemical staining of $\Delta \mathrm{MyK}$ (Fig. $5 \mathrm{C}$ ) or $\Delta+\mathrm{TK}$ testes (data not shown). Northern blot analysis corroborated that HSV thymidine kinase was not expressed in $\triangle M y K$ testes, and it is interesting that the 800-nucleotide transcript was also not expressed (Fig. 4A,B). Unfortunately, the $\Delta+$ TK line was lost before Northern blots of these transcripts could be assayed. It is unclear why HSV thymidine kinase was not expressed in the testes of $\Delta+\mathrm{TK}$ males because the MT-1 promoter and HSV thymidine 
Figure 7. MyK-103 rearrangements. Southern blots of DNA $(5 \mu \mathrm{g})$ from tail (males) or fetuses $(\mathrm{F})$ that was digested with PvuII. Two types of rearrangements, distinguished by their restriction and hybridization patterns, have been transmitted through the male germ line. (A) The type 1 rearrangement was transmitted to $G_{1}$ offspring by six $\mathrm{G}_{0}$ males (see Table 1), three of which are shown here, and to $G_{2}$ offspring in the $\Delta$ MyK line $\left(\mathrm{G}_{0}\right.$ male $\left.32-3\right)$. Each $\mathrm{G}_{0}$ male inherited the prototype MyK-103 transgene from its mother, evident by the restriction pattern in somatic (tail) DNA, but transmitted the type 1 rearrangement to its $G_{1}$

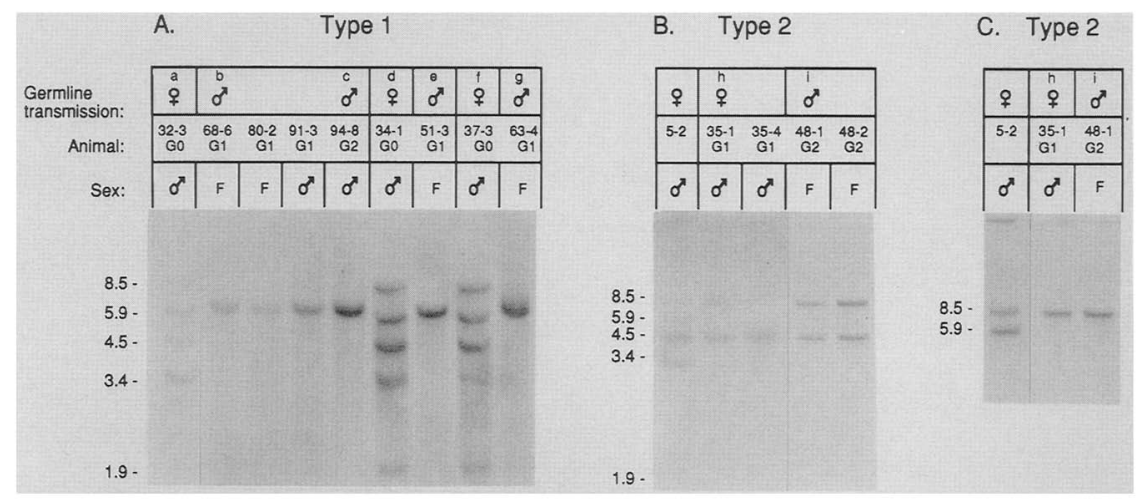
offspring. The filters were hybridized with pBR322. (B) The type 2 rearrangement was transmitted through two generations in the $\Delta+$ TK line. DNA from a hemizygous MyK-103 mouse was included for comparison. The filter was hybridized with pBR322. $(C)$ Same as $B$ but hybridized with the TK probe (see Fig. 1). The type 1 rearrangement hybridized only to pBR322 but the type 2 rearrangement also hybridized to the TK probe (8.5-kb band), although HSVtk protein was not expressed. Markers indicate the sizes of the PvuII restriction fragments from the prototype MyK-103 transgene; the 4.5-kb fragment spans the novel junction $\mathrm{J} 2$ and the $8.5-\mathrm{kb}$ fragment spans the novel junctions at $\mathrm{J5}, \mathrm{J6}$, and $\mathrm{J} 7$; all other bands correspond to restriction fragments within the transgene (Wilkie and Palmiter 1987). Germ-line transmission to $\mathrm{G}_{0}, \mathrm{G}_{1}$, and $\mathrm{G}_{2}$ offspring from (lane $a$ ) 14-2; (lane $b$ ) 32-3; (lane $c$ ) 91-3; (lane $d$ ) 17-3; (lane $e$ ) 34-1; (lane f) 132-8; (lane g) 37-3; (lane h) 15-8; (lane i) 35-4.

kinase coding sequences are apparently intact; only the $3^{\prime}$ untranslated sequence is truncated in this copy of pMK, but well downstream (190 nucleotides) of the two poly(A) addition sites in the HSV thymidine kinase gene (see Wagner et al. 1981 and Fig. 7B). Nevertheless, male germ-line transmission of these rearranged transgenes strengthens the conclusion that infertility of transgenic sperm and their meiotic partners is a dominant trait in MyK-103 males that is caused by expression of HSV thymidine kinase, possibly in combination with the 800 nucleotide transcript.

\section{Discussion}

Male mice in the MyK-103 pedigree are fertile but do not transmit the prototype transgenic insert to their off-

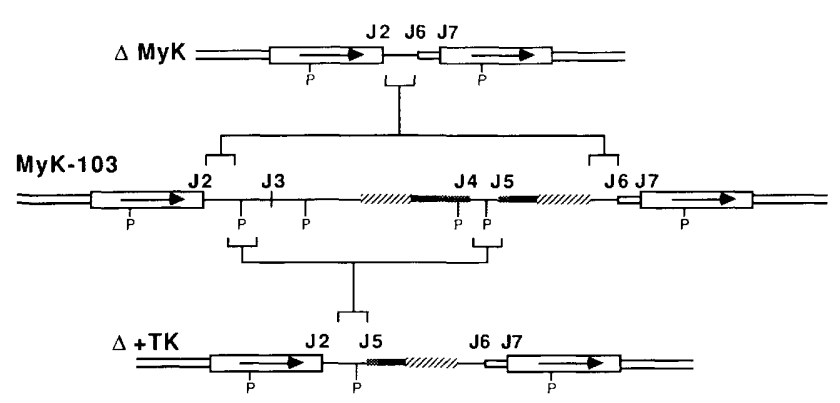

Figure 8. Derivation of $\Delta \mathrm{MyK}$ and $\Delta+\mathrm{TK}$. The structures of the type $1(\Delta \mathrm{MyK})$ and type $2(\Delta+\mathrm{TK})$ rearrangements of the prototype MyK-103 integrant were deduced by Southern blot analysis. $\triangle \mathrm{MyK}$ apparently arose by homologous recombination between two copies of pBR322 that were oriented in the same direction within the nucleotide sequence between positions 2823 and $3825 . \Delta+$ TK arose similarly by homologous recombination in pBR322 within the nucleotide sequence between positions 1204 and 2861 (see Wilkie and Palmiter 1987). spring. This phenomenon occurs because the germ line of every MyK-103 male is a genetic mosaic consisting of both transgenic and nontransgenic cells. The transgenic germ cells that retained the prototype insert expressed HSV thymidine kinase during the haploid phase of sperm development (Figs. 4 and 5), which apparently caused infertility of both transgenic sperm and their nontransgenic meiotic partners. MyK-103 males never sire offspring that inherit the prototype insert, and other lines of transgenic mice that expressed comparable levels of HSV thymidine kinase at the same stage of spermatogenesis are male sterile (Braun et al. 1990). In contrast to the other transgenic mice, MyK-103 males are fertile because the transgene is deleted in a significant proportion of the spermatogonia stem cells which, following spermatogenesis, give rise to fertile sperm.

Genetic analysis demonstrated that deletion of the transgene occurred by homologous recombination, most often between the duplicated $5-\mathrm{kb}$ sequence of mouse DNA that flanks either side of the insert (Fig. 3). Occasionally, internal rearrangements of the transgene, which were generated by homologous recombination between different fragments of pBR322 sequences in the prototype insert, were also transmitted through the male germ line (see Table 1). In these cases, either both copies of the HSV thymidine kinase gene present in the prototype MyK-103 insert were deleted (as for the type 1 rearrangement in the $\Delta \mathrm{MyK}$ line), or the single copy of the HSV thymidine kinase gene that remained in the type 2 rearrangement transmitted by the $\Delta+$ TK line (Fig. 8) did not express detectable amounts of protein. We inferred that the type 1 rearrangements occurred in the male germ line because each $G_{0}$ male inherited the prototype MyK-103 insert from his mother (Fig. 7A). The germ line in males $33-4,34-1,56-1$, and 57-1 was apparently a complex mosaic because the rearranged transgene was transmitted at low frequency (Table 1); most of the off- 
Table 1. Male germ-line transmission of transgene rearrangements

\begin{tabular}{|c|c|c|c|c|}
\hline & Male & Parent & Transgene & $\begin{array}{l}\text { Germ-line } \\
\text { transmission }\end{array}$ \\
\hline \multirow[t]{6}{*}{$\mathrm{G}_{0}$} & $32-3(\Delta \mathrm{MyK})$ & $14-2\langle F\rangle$ & type 1 & $26 / 70$ \\
\hline & $33-4$ & $132-10\langle F\rangle$ & type 1 & $2 / 17$ \\
\hline & $34-1$ & $17-3 \quad(\mathrm{~F})$ & type 1 & $1 / 11$ \\
\hline & $37-3$ & $132-8 \quad(F)$ & type 1 & $1 / 2$ \\
\hline & $56-1$ & $16-3 \quad(F)$ & type 1 & $1 / 11$ \\
\hline & $57-1$ & $17-3 \quad\{\mathrm{~F}\rangle$ & type 1 & $2 / 10$ \\
\hline \multirow[t]{2}{*}{$\mathrm{G}_{1}$} & $91-3(\Delta \mathrm{MyK})$ & $32-3 \quad(F)$ & type 1 & $13 / 26$ \\
\hline & $35-4(\Delta+\mathrm{TK})$ & $15-8 \quad(F)$ & type 2 & $3 / 7$ \\
\hline
\end{tabular}

Seven of 44 males in the MyK-103 pedigree have transmitted rearranged transgenes to their offspring. Six of these males are considered $\mathrm{G}_{0}$ founder mice of new sublines because they inherited the prototype MyK-103 insert from their mothers but have transmitted type 1 rearrangements to offspring. Male mice in the $\Delta \mathrm{MyK}$ transgenic line have transmitted a type 1 rearrangement for two generations. The type 2 rearrangement that was transmitted in the $\Delta+\mathrm{TK}$ line was inherited from female $15-8$ by male $35-1$ and his sibling $35-4$.

spring from these males probably inherited the meiotic products of germ cells that had fully deleted the MyK-103 insert. However, the majority of germ cells in these mice presumably retained the prototype insert, as evident, for example, in the comparable levels of HSV thymidine kinase RNA in the testes of 33-4 and other MyK-103 males (Fig. 4A). On the basis of the transmission frequency that we observed for the various recombination products, we presume that the germ line in most MyK-103 males is a four-component mosaic, consisting mostly of germ cells that inherit the prototype insert, some that inherit the complete deletion between the flanking repeats, and fewer still that inherit either the type 1 or type 2 rearrangement. The germ line of male $32-3$, who transmitted the type 1 rearrangement to nearly half of his offspring (Table 1), is an obvious exception to this general observation, presumably because this rearrangement occurred relatively early in germ cell development.

Due to the limitations of the genetic and immunological assays, we primarily detected recombination events that occurred in embryonic germ cells, prospermatogonia, and spermatogonial stem cells; homologous recombination events that might have occurred in germ cells committed to spermatogenesis were probably not detected. For example, if pMK were deleted during meiotic recombination, the resulting sperm would presumably still be infertile due to diffusion of HSV thymidine kinase from syncytial spermatids that developed from nonrecombinant spermatocytes (Fig. 5; see also Braun et al. 1989, 1990). By a similar argument, if deletion of pMK occurred in type A or type B spermatogonia, then only a fraction of the spermatids in a syncytium of this type would have expressed HSV thymidine kinase. As a result of diffusion within the syncytium, occasional patches of elongating spermatids might be recognized which stained very weakly with antibody against HSV thymidine kinase, but this was not observed. Further- more, we would expect pMK to be transmitted through the male germ line at some low frequency because other hemizygous transgenic mice that expressed low levels of HSV thymidine kinase, as in the $\mathrm{H}-40$ line reported by Braun et al. (1990), were fertile and transmitted the transgene to half of their offspring. However, not one of 636 offspring sired by 44 MyK-103 males has inherited the prototype MyK-103 insert. Immunocytochemistry revealed that almost all of the mosaic testicular tubules in the six hemizygous MyK-103 males that were analyzed had large patches of nontransgenic germ cells. The seminiferous tubules that were entirely devoid of HSV thymidine kinase provided evidence that deletion of pMK could occur during embryogenesis; that portion of the tubule containing only nontransgenic germ cells might have been colonized by nontransgenic prospermatogonia. This is the more likely explanation in mice that were sacrificed at a young age, but in older mice it is also possible that several independent deletion events occurred throughout adult life, eventually converting all of the spermatogonial stem cells in a given length of tubule to nontransgenic stem cells.

In MyK-103 testes, the proportion of seminiferous tubules that were mosaic or entirely devoid of HSV thymidine kinase ranged from $5 \%$ to $80 \%$, depending on the individual and his age. This high degree of mosaicism is probably required for fertility in MyK-103 males; an independent study showed that the threshold of fertility in male mice is at roughly $10 \%$ of the normal capacity for sperm production (Meistrich 1982). Given that fertile sperm only developed in those spermatid syncytia that did not express HSV thymidine kinase, we would expect MyK-103 males to be sterile if germ-line mosaicism was less pronounced. Germ-line mosaicism in MyK-103 males is significant in light of the fact that mosaicism was not detected in any of the sterile hemizygous males from the other transgenic lines that also expressed HSV thymidine kinase in spermatids (Braun et al. 1990). It is of further interest that 11 of the 16 founder males that were studied by Braun et al. (1990) were fertile but did not transmit the HSV thymidine kinase gene to their offspring; the remaining 5 males were each sterile. Immunocytochemical analysis of the fertile founder males revealed the mosaic expression of HSV thymidine kinase among spermatids, similar to the pattern seen in MyK-103 males. The fundamental difference was that these founder males were mosaic due to late integration of the transgene during embryogenesis (Wilkie et al. 1986), whereas mosaicism in hemizygous MyK-103 males was due to deletion of the transgene via homologous recombination.

Duplicated sequences and tandem repeats of genes and repetitive elements are inherently unstable in the genome. Several genetic diseases in humans are caused by deletions due to recombination between reiterated members of a multigene family, as observed in the globin locus (Higgs et al. 1989) and the red and green visual pigments (Nathans et al. 1986). Deletion breakpoints have also been identified between different $A l u$ sequences in the LDL receptor gene (Lehrman et al. 1987), and frequently the entire steroid sulfatase gene is 
deleted by recombination between flanking repeats in patients with ichthyosis (Ballabio et al. 1990; Yen et al. 1990). Although many deletions undoubtedly occur during meiosis, analysis of deletions in the Duchenne muscular dystrophy gene demonstrates that mitotic recombination does occur, resulting in germ-line mosaicism and the transmission of new mutations to offspring (Bakker et al. 1987; Darras and Francke 1987). Germ-line mosaicism was also found within certain repetitive DNA sequences of the VNTR family (Jeffreys et al. 1990).

A recombination rate for deletion of $\mathrm{pMK}$ from the germ line of MyK-103 males was estimated based on the extent of testicular mosaicism. To make this calculation, we assumed a constant recombination frequency per cell generation and that proliferation of transgenic stem cells was not under selection. Based on comparisons between other transgenic mouse lines, there is no evidence to suggest that the HSV thymidine kinase gene imparts any selective pressure on transgenic progenitor cells or stem cells (Wilkie et al. 1986; Heyman et al. 1989; Braun et al. 1990). Indeed, within the MyK-103 pedigree, the HSV thymidine kinase gene appears to be neutral during embryonic germ-line development prior to sexual dimorphism because females show no bias in germ-line transmission of $\mathrm{pMK}-148$ transgenic offspring among 315 born to hemizygous MyK-103 females. Within the male germ line of MyK-103 mice, we estimated a frequency of mitotic intrachromosomal recombination of about $2 \times 10^{-3}$ per cell division. Deletion of the transgene presumably occurred at a similar frequency in somatic cell lineages. Unfortunately, transcription of the HSV thymidine kinase gene is not prolific in somatic tissues of MyK-103 mice (Palmiter et al. 1984), which precludes an analysis of somatic mosaicism by immunocytochemistry. However, we documented one mosaic mouse among 148 transgenic offspring in the MyK-103 pedigree (Palmiter et al. 1984) that probably resulted from deletion of pMK early in embryogenesis (see Kelly et al. 1989). In contrast, somatic reversion at the murine $\mathrm{d}^{\mathrm{v}}$ locus, which occurred by intrachromosomal recombination between duplicated 520-bp LTRs, was detected once among 1.1 million mice (Seperack et al. 1988).

Mitotic recombination rates between duplicated sequences similar in length and structure to MyK-103 have been estimated in mouse somatic cell culture to be $2 \times 10^{-5}$ (Liskay et al. 1984) and in yeast to be between $2 \times 10^{-5}$ (Jackson and Fink 1981; Aguilera and Klein 1989) and $2 \times 10^{-4}$ (Hollingsworth and Byers 1989). Thus, the mitotic recombination frequency in the MyK-103 pedigree appears to be significantly greater than observed at other duplicated sequences of similar structure in eukaryotic cells, suggesting that MyK-103 integration locus contains a hotspot for homologous recombination. Most of the MyK-103 integrant and the flanking mouse DNA have been cloned, and it might be possible to reconstruct the pertinent elements of this region that would allow increased recombination frequency in tissue culture cells or transgenic mice. Furthermore, it should be possible to derive cell lines from
MyK-103 mice and use the selectable HSV thymidine kinase gene to study recombination frequencies in somatic cells.

\section{Materials and methods}

$D N A$ and RNA analysis

DNA manipulations and Southern blots were performed using standard procedures (Maniatis et al. 1982). Dot hybridization of tail DNA was performed as previously described (Brinster et al. 1985). RNA used for Northern blot analysis was extracted by homogenization in guanidinium isothiocyanate, followed by precipitation with lithium chloride as described (Cathala et al. 1983). RNA samples were electrophoresed in agarose-formaldehyde gels, transferred to nitrocellulose paper, and hybridized with radiolabeled probes as described (Peschon et al. 1987). All radiolabeled hybridization probes used in this study are indicated in Figure 1.

\section{Histology}

Histology was done on Carnoy's fixed tissues, which were embedded in paraffin, then sectioned and stained with hematoxylin and eosin or PAS. Staining for HSV thymidine kinase was done on paraffin-embedded sections using a rabbit anti-HSV thymidine kinase primary antibody and a peroxidase-AEC staining system.

\section{Calculation of the deletion frequency in the MyK-103 germ line}

To calculate the deletion frequency of pMK per cell division in the MyK-103 germ line, we chose a representative cross section of the testis from a hemizygous male, 92-1, which was sacrificed at 11 weeks of age and stained for testicular HSV thymidine kinase expression by immunocytochemistry. The sample was photographed at $40 \times$ magnification and a composite of the entire testis cross section was reconstructed (data not shown). In this section, 320 seminiferous tubules were displayed, 20 of which were mosaic or entirely lacked HSV thymidine kinase expression. The mosaic tubules were randomly distributed in the cross section. On average, $70 \%$ of the spermatids in these 20 tubules did not express HSV thymidine kinase. According to our assumptions (see text), the proportion of nonexpressing spermatids is equal to the proportion of spermatogonial stem cells that have deleted the transgene. Therefore, the fraction of recombinant spermatogonial stem cells (As-R) in the total stem cell population is calculated as $(0.7)(20) / 320=4.4 \%$ As-R. Assuming that As divide, on average, once every 8 days /the time required for one cycle of the seminiferous epithelium in mice; Oakberg 1971), then the elongating spermatids that were scored at 11 weeks were descendents of As cells that had undergone approximately nine stem cell divisions. Deletion of the transgene presumably occurs not only in spermatogonial stem cells but also during exponential growth of the primordial germ cells. A newborn mouse has approximately 40,000 spermatogonial stem cells $(20,000$ per testis; de Rooij 1983), which would be generated in 16 synchronous divisions of exponential growth. Neonatal germ cell death has not been assayed in mouse but, in rats, the embryonic testis contains about twice the number of precursor cells as later develop into spermatogonial stem cells (Beaumont and Mandl 1963).

The frequency of pMK deletion can be stated as the probability of a daughter cell inheriting a recombinant chromosome sometime during embryonic germ cell proliferation and spermatogonial stem cell renewal. The probability is calculated 
from the series

$$
P_{k}=\sum_{i=1}^{k} \mu(1-\mu)^{k-1}
$$

where $P_{k}$ is the probability that a cell is nontransgenic after $k$ divisions, and $\mu$ is the deletion rate per cell division. If $P_{k}$ is small, the deletion frequency is approximated as the percentage of nontransgenic spermatogonial stem cells divided by the number of generations in the stem cell lineage at the time the animal was sacrificed. The deletion frequency estimated from male $92-1$, whose germ-line was $4.4 \%$ mosaic when sacrificed at 11 weeks of age, was estimated to be $0.044 /(16+9)$, or $1.8 \times 10^{-3}$ per cell division. A similar calculation was made for male $33-4$, which had roughly $25 \%$ nontransgenic germ cells at 87 weeks of age, giving a frequency of $2.5 \times 10^{-3}$ per cell division.

\section{Acknowledgments}

We thank Charles Muller and Patricia Olds-Clarke for their interest in the early experiments on MyK-103 sperm fertility; Jesus del Mazo and Marvin Meistrich for their critical evaluation of our analysis; Jim Jaynes, Jeff Thorne, and Michael Strauss for suggesting the equation to calculate deletion probabilities, Michael Strathmann for discussions, and Kelwin Thomas for providing Northern blots of male germ cell RNA. We thank Kai Zinn, Anna Glascow, and Jim Jaynes for comments on the manuscript, and T.M.W. also thanks Mel Simon, in whose lab some of this work was accomplished. This work was funded, in part, by an National Institutes of Health (NIH) grant to R.D.P. (HD09172) and a NIH postdoctoral fellowship to T.M.W. (GM11576).

\section{References}

Aguilera, A. and H.L. Klein. 1989. Genetic and molecular analysis of recombination events in Saccharomyces cerevisiae occurring in the presence of the hyper-recombination mutation hprl. Genetics 122: 503-517.

Al-Shawi, R., J. Burke, C.T. Jones, J.P. Simons, and J.O. Bishop. 1988. A mup promoter-thymidine kinase reporter gene shows relaxed tissue-specific expression and confers male sterility upon transgenic mice. Mol. Cell. Biol. 8: 48214828.

Bakker, E., C. van Broeckhoven, E.J. Bonten, M.J. van de Vooren, H. Veenema, W. van Hul, G.J. van Ommen, A. Vanderberghe, and P.L. Pearson. 1987. Germline mosaicism and Duchenne muscular dystrophy mutations. Nature 329: 554-556.

Ballabio, A., B. Bardoni, S. Guioli, E. Basler, and G. Camerino. 1990. Two families of low copy number repeats are interspersed on $\mathrm{Xp22.3}$ : Implications for the high frequency of deletions in this region. Genomics (in press).

Beaumont, H.M. and A.M. Mandl. 1963. A quantitative study of primordial germ cells in the male rat. J. Embryol. Exp. Morphol. 11: 715-740.

Bennett, D. 1975. The T-locus of the mouse. Cell 6: 441-454.

Braun, R.E., R.R. Behringer, J.J. Peschon, R.L. Brinster, and R.D. Palmiter. 1989. Genetically haploid spermatids are phenotypically diploid. Nature 337: 373-376.

Braun, R.E., D. Lo, C.A. Pinkert, G. Widera, R.A. Flavell, R.D. Palmiter, and R.L. Brinster. 1990. Infertility in male trans- genic mice: Disruption of sperm development by HSV-tk expression in post meiotic germ cells. Biol. Reprod. 43: $684-693$.

Brinster, R.L., H.Y. Chen, M.E. Trumbauer, M.K. Yagle, and R.D. Palmiter. 1985. Factors affecting the efficiency of introducing foreign DNA into mice by microinjecting eggs. Proc. Natl. Acad. Sci. 82: 4438-4442.

Cathala, G., J.F. Savoret, B.L. Mendez, M. Karin, J.A. Martial, and J.D. Baxter. 1983. A method for isolation of intact, translationally active ribonucleic acid. DNA 2: 329-335.

Darras, B.T. and U. Francke. 1987. A partial deletion of the muscular dystrophy gene transmitted twice by an unaffected male. Nature 329: 556-558.

de Rooij, D.G. 1983. Proliferation and differentiation of undifferentiated spermatogonia in the mammalian testis. In Stem cells, their identification and characterization (ed. C. S. Potter), pp. 89-117. Churchill Livingstone, Edinburgh.

Dym, M. and D.W. Fawcett. 1971. Further observations in the number of spermatogonia, spermatocytes and spermatids connected by intercellular bridges. Biol. Reprod. 4 195-215.

Hartl, D.L. 1971. Recurrence risks for germinal mosaics. Am. J. Hum. Genet. 23: 124-134.

Hartl, D.L. and Y. Hirazumi. 1976. Segregation distortion. In The genetics and biology of Drosophila, $1 \mathrm{~b}$ (ed. M. Ashburner and E. Novitshi), pp. 616-666. Academic Press, New York.

Heyman, R.A., E. Borrelli, J. Lesley, D. Andersen, D.D. Richmann, J.M. Baird, R. Hyman, and R.M. Evans. 1989. Thymidine kinase obliteration: Creation of transgenic mice with controlled immune deficiency. Proc. Natl. Acad. Sci. 86: $2698-2702$.

Higgs, D.R., M.A. Vickers, A.O.M. Wilkie, I.M. Pretorius, A.P. Jarman, and D.J. Weatherall. 1989 A review of the molecular genetics of the human globin gene cluster. Blood 73: 10811104.

Hollingsworth, N.M. and B. Byers. 1989. HOP 1: A yeast meiotic pairing gene. Genetics 121: 445-462.

Iwakura, Y., M. Asano, Y. Nishimune, and Y. Kawade. 1988. Male sterility of transgenic mice carrying exogenous mouse interferon- $\gamma$ gene under the control of the metallothionein enhancer-promoter. EMBO I. 12: 3757-3762.

Jackson, J.A. and G.R. Fink. 1981. Gene conversion between duplicated genetic elements in yeast. Nature 292: 306-311.

Jeffreys, A.J., R. Neumann, and V. Wilson. 1990. Repeat unit sequence variation in minisatellites: A novel source of DNA polymorphism for studying variation and mutation by single molecule analysis. Cell 60: 473-485.

Kelly, R., G. Bulfield, A. Collick, M. Gibbs, and A.J. Jeffreys. 1989. Characterization of a highly unstable mouse minisatellite locus: Evidence for somatic mutation during early development. Genomics 5: 844-856.

Lehrman, M.A., J.L. Goldstein, D.W. Russell, and M.S. Brown. 1987. Duplication of seven exons in LDL receptor gene caused by Alu-Alu recombination in a subject with familial hypercholesterolemia. Cell 48: 287-835.

Liskay, R.M., J.L. Stachelek, and A. Letsou. 1984. Homologous recombination between repeated chromosomal sequences in mouse cells. Cold Spring Harbor Symp. Quant. Biol. 49: $183-189$.

Maniatis, T., E.F. Fritsch, and J. Sambrook. 1982. Molecular cloning: A laboratory manual. Cold Spring Harbor Laboratory, Cold Spring Harbor, New York.

Meistrich, M.L. 1982. Quantitative correlation between testicular stem cell survival, sperm production, and fertility in the mouse after treatment with different cytotoxic agents. $J$. Androl. 3: 58-68. 
Wilkie et al.

Nathans, J., T.P. Piantanida, R.L. Eddy, T.B. Shows, and D.S. Hogness. 1986. Molecular genetics of ingerited variation in human color vision. Science 232: 203-210.

Oakberg, E.F. 1971. Spermatogonial stem cell renewal in the mouse. Anat. Rec. 169: 515-530.

Palmiter, R.D., H.Y. Chen, and R.L. Brinster. 1982. Differential regulation of metallothionein-thymidine kinase fusion genes in transgenic mice and their offspring. Cell 29: 701710.

Palmiter, R.D., T.M. Wilkie, H.Y. Chen, and R.L. Brinster. 1984. Transmission distortion and mosaicism in an unusual transgenic mouse pedigree. Cell 36: 869-877.

Peschon, J.J., R.R. Behringer, R.L. Brinster, and R.D. Palmiter. 1987. Spermatid-specific expression of protamine 1 in transgenic mice. Proc. Natl. Acad. Sci. 84: 5314-5319.

Seperack, P.K., M.C. Strobel, D.J. Corrow, N.A. Jenkins, and N.G. Copeland. 1988. Somatic and germ-line reverse mutation rates of the retrovirus-induced dilute coat-color mutation of DBA mice. Proc. Natl. Acad. Sci. 85: 189-192.

Thomas, K., T.M. Wilkie, P. Tomashefski, A. Bellvedee, and M.I. Simon. 1989. Differential gene expression during mouse spermatogenesis. Biol. Reprod. 41: 729-739.

Wagner, M.J., J.A. Sharp, and W.C. Summers. 1981. Nucleotide sequence of the thymidine kinase gene of herpes simplex virus type 1. Proc. Natl. Acad. Sci. 78: 1441-1445.

Wilkie, T.M. and R.D. Palmiter. 1987. Analysis of the integrant in MyK-103 transgenic mice in which males fail to transmit the integrant. Mol. Cell. Biol. 7: 1646-1655.

Wilkie, T.M., R.L. Brinster, and R.D. Palmiter. 1986. Germline and somatic mosaicism in transgenic mice. Dev. Biol. 118: $9-18$.

Yen, P.H., X.-M. Li, S.-P. Tsai, C. Johnson, T. Mohandas, and L.J. Shapiro. 1990. Frequent deletions of the human X chromosome distal short arm result from recombination between low copy repetitive elements. Cell 61: 603-610. 


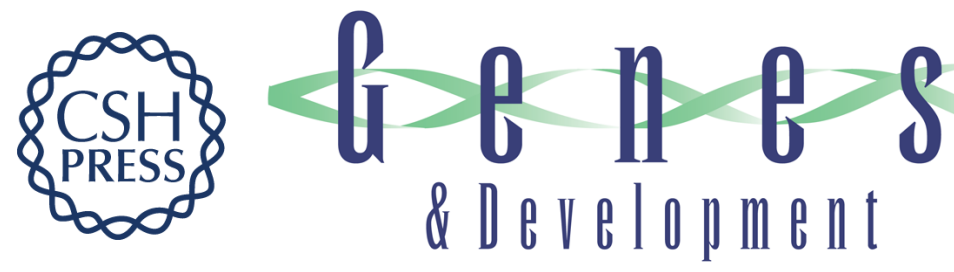

\section{Germ-line intrachromosomal recombination restores fertility in transgenic MyK-103 male mice.}

T M Wilkie, R E Braun, W J Ehrman, et al.

Genes Dev. 1991, 5:

Access the most recent version at doi:10.1101/gad.5.1.38

References This article cites 33 articles, 11 of which can be accessed free at:

http://genesdev.cshlp.org/content/5/1/38.full.html\#ref-list-1

License

Email Alerting Receive free email alerts when new articles cite this article - sign up in the box at the top Service right corner of the article or click here.

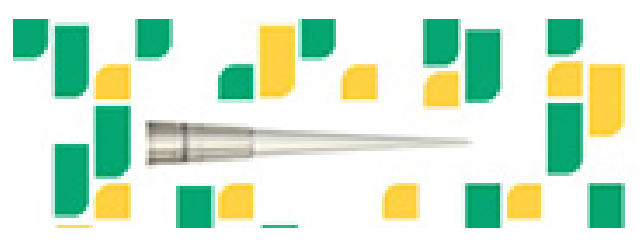

Focused on your science. 